\title{
Actualización y combinación de las guías de cuidados preventivos de la fuerza de tareas de EEUU y Canadá (primera parte: Consejería)
}

Combination and update of preventive care guidelines of the U.S. and Canadian task forces (first part: Counseling)

Agustín Ciapponi*

\begin{abstract}
Resumen
La Fuerza de Tareas Preventiva de los EE.UU. (sigla en inglés: USPSTF) y la Fuerza de Tareas Canadiense de Cuidados Preventivos de la Salud (sigla en inglés: CTFPHC) son las dos instituciones más importantes del mundo en la evaluación de cuidados preventivos. Ambas emiten y actualizan constantemente recomendaciones preventivas; y las mismas pueden accederse en forma completa y gratuita a través de sus sitios Web: (http://www.ahrq.gov/clinic/cps3dix.htm, y http://www.ctfphc.org). En esta primera entrega ofrecemos una actualización de las prácticas referidas al consejo preventivo y su justificación clínica emitida por ambas entidades, respecto de aquellas previamente resumidas en el $2003^{1}$ y en el $2005 / 6^{2,3}$, en EVIDENCIA. Estas habían alcanzado las recomendaciones generadas hasta septiembre de 2005; lo cual implica la actualización o incorporación de aquellas recomendaciones aquí publicadas con fecha posterior.

No se incluyen en esta revisión prácticas como inmunizaciones ni de profilaxis postexposición de enfermedades infecciosas, pues estas se rigen habitualmente por normas locales como las Normas Nacionales de Vacunación. Muchas recomendaciones emitidas hace varios años se encuentran bajo revisión pero las mantenemos a título informativo hasta que los cambios sean confirmados.

\section{Abstract}

The US Preventive Services Task Force (USPSTF) and the Canadian Task Force on Preventive Health Care (CTFPHC) are the two most important institutions in the world in the evaluation of preventive care. Both released and updated preventive recommendations, which can be accessed in full and free through its Web sites: (http://www.ahrq.gov/clinic/cps3dix.htm and http://www.ctfphc.org ). In this first issue we offer an update on preventive counseling and its clinical justification issued by both entities. Practices as immunizations and post exposure prophylaxis of infectious diseases are not included in this review, as these are usually governed by local rules. Many recommendations made several years ago are under review but we keep them until the changes are confirmed.
\end{abstract}

Palabras clave: guía de práctica clínica, consejo preventivo. Key words: clinical practice guideline, preventive counseling.

Ciapponi A. Actualización y combinación de las guías de cuidados preventivos de las fuerzas de tareas de EE.UU. y Canadá.(Primera parte: Consejería). Evid. Act. Pract. Ambul. 12(2). 61-65. Abr-Jun. 2009

Calidad de la evidencia y grados de recomendación En cuanto a la valoración de la calidad de la evidencia y la fuerza de las recomendaciones, cabe mencionar que la USPSTF ha actualizado su nomenclatura en mayo de 2007, para incorporar "niveles de certeza" en sus recomendaciones (pro-babilidad de que el beneficio neto evaluado por la USPSTF sea correcto) y "sugerencias para la práctica" (ver tabla 1$){ }^{4}$

En esta entrega decidimos incorporar adicionalmente el sistema GRADE. El grupo de trabajo GRADE (Grading of Recommendations Assessment, Development and Evaluation, http://www.gradeworkinggroup.org/_ES/index.htm) comenzó en el año 2000 con el objetivo de abordar la heterogeneidad y las amplias deficiencias de los actuales sistemas de clasificación de la evidencia. Este grupo de trabajo ha elaborado una clasificación con un enfoque sistemático y explícito, a partir de la definición de una serie de criterios necesarios para hacer juicios sobre la calidad de la evidencia y la fuerza de las recomendaciones que apunta convertirse el sistema de mayor consenso internacional ${ }^{5,6}$. La CTFPHC adhirió a este sistema desde 2003. Aunque en el sitio Web solo se aplicó prospectivamente a partir de esa fecha, a los fines de facilitar la comprensión la antigua clasificación fue actualizada en esta publicación. En el sistema GRADE existen dos conceptos centrales ${ }^{7}$ que intentamos correlacionar con los de la clasificación de la USPSTF en la tabla y figura 1 :

\section{Calidad de la evidencia}

Se refiere al grado de confianza que se puede tener en que la estimación de un efecto (en un determinado estudio) es adecuada. Puede subdividirse en: Alta $(\oplus \oplus \oplus \oplus)$, Moderada $(\oplus \oplus \oplus \mathrm{O})$, Baja $(\oplus \oplus \mathrm{OO})$ o Muy Baja $(\oplus \mathrm{OOO})$.

Los ensayos clínicos aleatorizados corresponden al nivel de calidad mas alto posible; existiendo criterios, que a su vez, pueden bajar su puntuación. Por otro lado, los estudios observacionales, corresponden a un nivel de calidad bajo; a su vez existen criterios que pueden subir la puntuación de los mismos. Otros tipos de diseños corresponden a estudios de calidad muy baja.

\section{Grado de recomendaciones}

Refleja hasta que punto podemos confiar, para la población en la que se aplicará una determinada guía, en que la puesta en práctica de la recomendación conllevará más beneficios que riesgos. En la clasificación GRADE, esta categorización resulta simplificada, con recomendaciones fuertes y débiles (ambas pueden ser a favor o en contra de una determinada intervención). Una recomendación es fuerte (I) si considera que más del $90 \%$ de las personas elegirían esa opción y débil (II) si considera que entre 51 y $91 \%$ de las personas elegirían esa opción. Con cifras menores no efectúa recomendaciones.

Figura 1: resumen de la clasificación de las prácticas preventivas según la evidencia que la sustenta y la fuerza de la recomendación para realizarla.

\begin{tabular}{|c|c|c|c|}
\hline \multicolumn{4}{|c|}{ Recomendaciones USPSTF (GRADE) } \\
\hline A (I) & B (II) & C (-) & D (I) \\
\hline $\begin{array}{l}\text { Realizar } \\
\text { 3enefico }\end{array}$ & & & $\begin{array}{l}\text { No } \\
\text { realizar }\end{array}$ \\
\hline $\begin{array}{c}\text { Evidencia } \\
\text { Buena }\end{array}$ & & & $\begin{array}{l}\text { Evidencia } \\
\text { Buena }\end{array}$ \\
\hline
\end{tabular}


Tabla 1: nivel de recomendación de prácticas preventivas

\begin{tabular}{|c|c|c|c|c|}
\hline \multicolumn{3}{|c|}{ USPSTF } & \multicolumn{2}{|c|}{ GRADE* } \\
\hline Tipo & Definición & Sugerencias para la práctica & $\begin{array}{l}\text { Calidad de la } \\
\text { Evidencia }\end{array}$ & $\begin{array}{l}\text { Grado de } \\
\text { Recomendación }\end{array}$ \\
\hline A & $\begin{array}{l}\text { Recomienda fuertemente el servicio. Existe alta } \\
\text { evidencia } \pm \text { de que el beneficio neto es sustancial. }\end{array}$ & Ofrecer o proveer el servicio. & $\begin{array}{l}\text { Alta } \\
\oplus \oplus \oplus \oplus\end{array}$ & I (Fuerte) \\
\hline B & $\begin{array}{l}\text { Recomienda el servicio. Existe alta certeza de que el } \\
\text { beneficio neto es moderado, o hay moderada evidencia } \\
\text { de que el beneficio neto es moderado a sustancial. }\end{array}$ & Ofrecer o proveer el servicio. & $\begin{array}{l}\text { Moderada } \\
\oplus \oplus \oplus \mathrm{O}\end{array}$ & II (Débil) \\
\hline $\mathbf{C}$ & $\begin{array}{l}\text { Recomienda no proveer el servicio de manera rutinaria, } \\
\text { aunque es opcional y puede haber consideraciones en } \\
\text { casos individuales. Existe evidencia al menos moderada } \\
\text { de que el beneficio neto es pequeño. }\end{array}$ & $\begin{array}{l}\text { Ofrecer } 0 \text { proveer el servicio solo si otras } \\
\text { consideraciones lo apoyan en el paciente individual. }\end{array}$ & $\begin{array}{l}\text { Moderada } \\
\oplus \oplus \oplus \mathrm{O}\end{array}$ & - \\
\hline D & $\begin{array}{l}\text { Recomienda no proveer el servicio. Existe evidencia } \\
\text { moderada a alta de que el servicio no provee un } \\
\text { beneficio neto, o que los daños superan los beneficios. }\end{array}$ & Desaconsejar la provisión del servicio. & $\begin{array}{l}\text { Moderada/Alta } \\
\oplus \oplus \oplus \oplus^{*}\end{array}$ & I (Fuerte) \\
\hline I & $\begin{array}{l}\text { La evidencia es Insuficiente, de mala calidad, o no } \\
\text { puede determinarse el balance entre beneficios y daños } \\
\text { por lo que no puede hacerse una recomendación. }\end{array}$ & $\begin{array}{l}\text { Si se ofrece el servicio, el paciente debe comprender } \\
\text { la incertidumbre alrededor del balance riesgo beneficio. }\end{array}$ & $\begin{array}{l}\text { Baja/Muy baja } \\
\qquad \oplus \oplus^{*} \mathrm{OO}\end{array}$ & - \\
\hline
\end{tabular}

Adaptado de: U.S. Preventive Services Task Force Grade Definitions. May 2008. Agency for Healthcare Research and Quality, Rockville, MD.

\pm Un nivel de evidencia alto implica que incluye resultados consistentes provenientes de estudios bien conducidos en una población representativa; y que es poco probable que las conclusiones sean afectadas por resultados de futuras investigaciones. Un nivel moderado implica que la consistencia de los resultados esta debilitada y es posible que la dirección del efecto observado pueda cambiar a la luz de nuevos estudios. Un nivel bajo implica que su calidad es claramente insuficiente y que se requerirá más información para permitir una mejor estimación de los efectos de la practica.

El \# indica que algunos componentes de la recomendación están basados también en un nivel inferior de evidencia. Por ejemplo $\oplus \oplus \#$ OO significa que una recomendación está basada en niveles $\oplus$ OOO y $\oplus \oplus$ OO.

Tabla 2: resumen de los principales consejos preventivos según la USPSTF, con aportes complementarios de la CTFPHC.

\begin{tabular}{|c|c|c|c|c|}
\hline \multirow[b]{2}{*}{ Condición clínicá } & \multirow[b]{2}{*}{ Práctica preventiva } & \multicolumn{2}{|c|}{ Recomendación } & \multirow{2}{*}{$\begin{array}{l}\text { Fecha de } \\
\text { publicación }\end{array}$} \\
\hline & & USPSTF & Grade & \\
\hline Cáncer ginecológico & Consejo sobre medidas de prevención primaria & 1 & $-\oplus \oplus \# \mathrm{OO}$ & 1996 \\
\hline Cáncer de piel & Consejo para reducir la exposición solar provisto por médicos de atención primaria & $I^{*}$ & $-\oplus \oplus \# \mathrm{OO}$ & $10 / 2003$ \\
\hline \multirow[t]{2}{*}{$\begin{array}{l}\text { Enfermedades crónicas } \\
\text { relacionadas a la dieta }\end{array}$} & $\begin{array}{l}\text { Consejo de intensidad media sobre dieta saludable dirigido a pacientes no seleccionados } \\
\text { en atención primaria }\end{array}$ & I & $-\oplus \oplus \# \mathrm{OO}$ & \multirow[t]{2}{*}{$1 / 2003$} \\
\hline & $\begin{array}{l}\text { Consejo de intensidad media-alta sobre dieta saludable dirigido a adultos con dislipidemia } \\
\text { y riesgo de enfermedades cardiovasculares }\end{array}$ & $\mathrm{B}^{\star}$ & $\mathrm{II} \oplus \oplus \oplus \mathrm{O}$ & \\
\hline \multirow{4}{*}{$\begin{array}{l}\text { Sobrepeso } \\
\text { y } \\
\text { obesidad }^{\text {CTF }}\end{array}$} & Consejo sobre programas intensivos de dieta hipocalórica/hipograsa & B & $\mathrm{II} \oplus \oplus \oplus \mathrm{O}$ & \multirow{4}{*}{2006} \\
\hline & $\begin{array}{l}\text { Promoción del ejercicio y la dieta hipocalórica/hipograsa mediante intervenciones de baja } \\
\text { intensidad administrados por teléfono o correo electrónico, o empleando incentivos } \\
\text { financieros }\end{array}$ & $\mathrm{D}$ & $\mathrm{I} \oplus \oplus \oplus \oplus^{\#}$ & \\
\hline & Programa de fortalecimiento & C & $-\oplus \oplus \# \mathrm{OO}$ & \\
\hline & Intervenciones comunitarias de prevención de enfermedad cardiovascular & I & $-\oplus \oplus \# \mathrm{OO}$ & \\
\hline \multirow[t]{2}{*}{ Abuso de alcohol } & Consejo en adultos, incluso en embarazadas, en ámbitos de atención primaria & B & $\mathrm{II} \oplus \oplus \oplus \mathrm{O}$ & \multirow[b]{2}{*}{$4 / 2004$} \\
\hline & Consejo en adolescentes en ámbitos de atención primaria & I & $-\oplus \oplus \# \mathrm{OO}$ & \\
\hline \multirow[t]{2}{*}{ Lesiones no intencionales } & Consejo preventivo de accidentes en el hogar y accidentes recreacionales & $\begin{array}{l}\text { B } \\
(I)^{*}\end{array}$ & $\begin{array}{l}\mathrm{II} \oplus \oplus \oplus \mathrm{O} \\
-\oplus \oplus \# \mathrm{OO}\end{array}$ & \multirow{2}{*}{1996} \\
\hline & Consejo a pacientes y padres de jóvenes sobre accidentes vehiculares & $\mathrm{B}$ & $\mathrm{II} \oplus \oplus \oplus \mathrm{O}$ & \\
\hline \multirow[t]{2}{*}{ Tabaquismo } & Consejo de cese tabáquico en adultos y embarazadas & A & $\mathrm{I} \oplus \oplus \oplus \oplus$ & $4 / 2009$ \\
\hline & Consejo preventivo o de cese tabáquico en niños y adolescentes & I & $-\oplus \oplus \# \mathrm{OO}$ & $11 / 2003$ \\
\hline \multirow[t]{2}{*}{$\begin{array}{l}\text { Enfermedades de transmisión } \\
\text { sexual (ETS) }\end{array}$} & $\begin{array}{l}\text { Consejo conductual de alta intensidad en adolescentes sexualmente activos y adultos en } \\
\text { riesgo }\end{array}$ & B & $\mathrm{II} \oplus \oplus \oplus \mathrm{O}$ & \multirow[t]{2}{*}{$10 / 2008$} \\
\hline & Consejo en adolescentes sexualmente inactivos y adultos sin riesgo & I & $-\oplus \oplus \# \mathrm{OO}$ & \\
\hline Enfermedades crónicas & Consejo de actividad física en atención primaria & I & $\mathrm{II} \oplus \oplus \oplus \mathrm{O}$ & 2002 \\
\hline Embarazo no des eado & Consejo a hombres y mujeres en riesgo de sufrirlo & B & $\mathrm{II} \oplus \oplus \oplus \mathrm{O}$ & 1996 \\
\hline Violencia juvenil & Consejo a adolescentes y adultos asintomáticos & I & $-\oplus \oplus \# \mathrm{OO}$ & 1996 \\
\hline Llanto nocturno ${ }^{\text {CTF }}$ & Consejo anticipatorio para padres & A & $\mathrm{I} \oplus \oplus \oplus \oplus$ & 1994 \\
\hline Dolor de espalda & Consejo sobre intervenciones preventivas en adultos en atención primaria & I & $-\oplus \oplus \# \mathrm{OO}$ & $2 / 2004$ \\
\hline $\begin{array}{l}\text { Enfermedades relacionadas a la } \\
\text { ausencia de lactancia }\end{array}$ & Promoción de la lactancia durante y después del embarazo & B & $\mathrm{II} \oplus \oplus \oplus \mathrm{O}$ & $10 / 2008$ \\
\hline
\end{tabular}

Los aportes complementarios de la CTFPHC a la USPSTF se identificaron con CTF en superíndice sobre la recomendación.

Un * sobre el grado de recomendación indica que éste puede modificarse bajo ciertas circunstancias explicadas en el apartado correspondiente o por observaciones hechas por la CTFPHC. El \# indica que algunos componentes de la recomendación están basados también en un nivel inferior de evidencia. Por ejemplo $\oplus \oplus \# \mathrm{OO}$ significa que una recomendación está basada en niveles $\oplus$ OOO y $\oplus \oplus \mathrm{OO}$. 


\section{Cáncer}

Cáncer ginecológico. Consejo sobre medidas de prevención primaria (I)

Los consejos médicos sobre anticoncepción deberían incluir información de los beneficios potenciales de los anticonceptivos orales, métodos de barrera y ligadura de trompas con respecto a su relación con los cánceres ginecológicos. Asimismo los médicos deben promover otras prácticas preventivas de probados beneficios en salud, que también pueden reducir la incidencia de cánceres ginecológicos, como el mantenimiento de un adecuado peso corporal, el cese tabáquico y la practica de sexo seguro. La evidencia resulta insuficiente como para expedirse a favor o en contra del consejo de rutina para la prevención primaria de cánceres ginecológicos

\section{Cáncer de Piel. Consejo provisto por médicos de atención} primaria para reducir la exposición solar (I)

El consejo a padres puede incrementar el uso de pantallas protectoras en niños pero es insuficiente la evidencia sobre modificación de otras conductas (ropas protectoras, reducir la exposición solar, evitar el uso de lámparas/camas solares o el autoexamen de piel) y sobre sus posibles daños.

La CTFPHC recomienda aconsejar a la población general evitar la exposición solar en horas del medio día, así como el uso de vestimenta protectora (B). Por otro lado no se expide ni a favor ni en contra sobre el uso de pantallas solares (C); si bien esta última posiblemente sea "A" dada la nueva evidencia. La evidencia actual resulta insuficiente como para determinar si el consejo médico es efectivo en modificar conductas de los pacientes para reducir el riesgo de cáncer de piel.

\section{Enfermedades crónicas relacionadas a la dieta, sobrepeso y obesidad}

La CTFPHC actualizó en el 2006 las recomendaciones publicadas por la USPSTF en 12/2003. Estas habían hallado insuficiente evidencia como para recomendar a favor o en contra intervenciones de intensidad baja o media e intervenciones conductuales para descenso sostenido de peso en adultos obesos (índice de masa corporal o IMC mayor a $30 \mathrm{~kg} / \mathrm{m}^{2}$ ) o con sobrepeso (IMC de 25 a $29,9 \mathrm{~kg} / \mathrm{m}^{2}$ ).

Consejo de intensidad media sobre dieta saludable dirigido a pacientes no seleccionados en atención primaria (I)

Se encontró evidencia aceptable de que en ámbitos de atención primaria el consejo dietético breve, de intensidad media puede producir de pequeños a moderados cambios en el consumo promedio diario de los componentes básicos de una dieta saludable (especialmente grasas saturadas, frutas y vegetales). La fuerza de esta evidencia, sin embargo, es limitada pues confía en auto-reportes de dieta, en un uso limitado de medidas que corroboran cambios de dieta, en seguimientos limitados (no más allá de seis a doce meses) y en participantes de estudios que podrían no ser completamente representativos de pacientes de atención primaria. Además, hay evidencia limitada como para determinar posibles daños. Se concluyó que hay escasa evidencia para determinar la significancia y magnitud de los beneficios del consejo para promover una dieta sana en adultos. Aunque hay estudios basados en la comunidad que han evaluado medidas para reducir el consumo de grasas en niños, no se identificaron ensayos controlados que intervengan sobre el consejo dietético en niños o adolescentes en atención primaria.

Consejo de intensidad media-alta sobre dieta saludable dirigido a adultos con dislipidemia y riesgo de enfermedades cardiovasculares (B)

Se encontró buena evidencia de que el consejo de intensidad media a alta dirigido a adultos en riesgo de sufrir enfermedades crónicas relacionadas a la dieta, puede producir cambios moderados a grandes en el consumo promedio diario de los componentes básicos de una dieta sana (incluyendo grasas saturadas, fibras, frutas y vegetales). Esta evidencia (proveniente de ensayos controlados) combina la educación nutricional con el consejo sobre comportamiento dietético proporcionado por nutricionistas, dietistas, o profesionales de atención primaria entrenados (ej. médicos o enfermeras). No se identificaron ensayos controlados sobre consejo dietético intensivo en niños o adolescentes. La CTFPHC recomienda aconsejar el consumo de vegetales de hoja verde y frutas en fumadores (B). Es probable que el consejo mejore los resultados de salud y que los beneficios compensen los daños potenciales.

\section{Consejo sobre programas intensivos de dieta hipocalóri-} $\mathrm{ca} /$ hipograsa (B)

El consejo individual intensivo y de pequeños grupos mostró efectos positivos en el peso corporal y en los niveles de colesterol en hombres con sobrepeso. La CTFPHC concluye que existe evidencia aceptable como para recomendar el consejo individual intensivo y de pequeños grupos de dieta hipocalórica o hipograsa para prevenir la obesidad (B).

Promoción del ejercicio y la dieta hipocalórica/hipograsa mediante intervenciones de baja intensidad administrados por teléfono o correo electrónico, o empleando incentivos financieros (D)

Las intervenciones educativas dirigidas a adultos con sobrepeso fallan en reducir el peso, aunque favorecen algunos cambios de comportamiento relacionados. La CTFPHC concluye que existe evidencia suficiente como para no aconsejar dichas intervenciones.

\section{Programa de fortalecimiento (C)}

Un programa de entrenamiento de resistencia en mujeres reduce la grasa corporal pero no el peso ni la circunferencia de cintura. Hay buena evidencia pero los resultados son inciertos como para recomendar un programa de fortalecimiento para prevenir la obesidad.

Intervenciones comunitarias de prevención de enfermedad cardiovascular para prevenir la obesidad (I)

Los programas que promueven el ejercicio, dieta saludable, antropometría, programas de descenso de peso en el trabajo, enlentecen la ganancia de peso pero no previenen la obesidad. Hay insuficiente evidencia como para recomendar a favor o en contra de programas preventivos comunitarios de enfermedad cardiovascular para prevenir la obesidad

\section{Abuso de alcohol}

Consejo en adultos, embarazadas (B) y adolescentes (I) en ámbitos de atención primaria

Las intervenciones iniciales incluyen un consejo inicial de 15 minutos, feedback, consejo y fijar metas. La mayoría incluyen mayor asistencia, intervenciones motivacionales y seguimiento. La evidencia sostiene además que estos efectos positivos perduran hasta cuatro o más años luego de la intervención. Sin embargo hay limitad evidencia de que el rastreo y el consejo conductual reduzcan la morbilidad por alcohol.

Existe evidencia aceptable de que en adultos el consejo conductual breve con seguimiento posterior reduce leve a moderadamente el consume de alcohol por 6-12 meses o más.

La evidencia de la efectividad del consejo para reducir el consumo de alcohol durante el embarazo es limitada, sin embargo los estudios en población general adulta muestran que el 
consejo conductual es efectivo en mujeres que amamantan. Las embarazadas deben ser informadas de los efectos nocivos del alcohol sobre el feto y debe aconsejarse la abstinencia. En estas poblaciones los beneficios del consejo conductual para reducir el abuso de alcohol superan los daños potenciales. La evidencia en ámbitos de atención primaria es insuficiente como para valorar los potenciales beneficios y daños del rastreo y consejo conductual en adolescentes pero si lo es en adultos y embarazadas.

\section{Lesiones traumáticas no intencionales (ex "accidentes") Consejo para prevenir las lesiones no intencionales hoga- reñas y durante la recreación (B)}

Algunos de los consejos incluyen: prevención de quemaduras, caídas, ingestión de objetos pequeños, intoxicaciones, lesiones con objetos puntiagudos o filosos; la instalación de disyuntor de corriente eléctrica; la educación sobre la peligrosidad de los automóviles, el fuego, de arrojar objetos y de subirse a cornisas, techos y árboles; el resguardo de armas de fuego e iniciación en la práctica de la natación. Si bien esta recomendación esta basada en términos de eficacia (B), no lo es así en términos de efectividad(I).

Las personas con problemas de alcohol o drogas deberían ser identificadas, aconsejadas y seguidas, a fin de evitar actividades potencialmente peligrosas mientras estén intoxicadas. Se recomienda aconsejar a los pacientes mayores sobre medidas específicas para prevenir las caídas (B), aunque también en estos casos la efectividad del consejo no se ha evaluado adecuadamente (I). En pacientes mayores de alto riesgo (en ámbitos que cuenten con los recursos adecuados a tal fin) se recomienda una intervención multifactorial individualizada más intensiva. Por otro lado, existe evidencia insuficiente para recomendar a favor o contra el uso de protectores de la cadera externos para prevenir las lesiones por caídas (I). Se recomienda el consejo periódico a los padres para reducir el riesgo de accidentes en el hogar y accidentes recreacionales de sus niños y aconsejar medidas para prevenir caídas en adultos mayores.

La CTFPHC establece al respecto para pacientes adultos: evidencia aceptable para recomendar la legislación sobre uso de casco al andar en bicicleta en calles y la prohibición del consumo de alcohol al navegar o practicar deportes acuáticos (B); e insuficiente evidencia a favor o en contra del consejo individual para ambas actividades $(C)$. Tampoco existe evidencia suficiente para expedirse a favor o en contra de la legislación y/o consejo individual sobre mantener inaccesibles las armas de fuego cargadas y sobre el uso de la maniobra de Heimlich para tratar la obstrucción de la vía aérea por cuerpo extraño (C).

En cuanto a las recomendaciones dirigidas a niños y adolescentes se establece: que existe evidencia aceptable para recomendar la implementación de legislación y consejo individual sobre el uso de protección en ventanas y escaleras para evitar caídas (B). Que existe evidencia aceptable para recomendar la implementación de legislación y educación pública sobre enseñanza a los niños de los peligros relacionados al agua, impartir clases de natación, recomendar el uso de caso al andar en bicicleta y no dejar nunca solos en la bañadera a niños menores de 3 años (B). Que existe evidencia aceptable para recomendar la implementación de legislación, educación pública y consejo individual sobre uso de dispositivos detectores de humo, pijamas hechos de tela no inflamable, y calentadores de agua caliente regulados por termostato para evitar quemaduras (B); y buena evidencia para recomendar el uso de jarabe de ipecacuana y la implementación de programas regionales de vigilancia para el tratamiento de intoxica- ciones (A para legislación, educación y consejo, y B solo para el consejo individual). Por otro lado, no existe evidencia suficiente para recomendar a favor o en contra sobre el consejo individual de enseñar a los niños sobre los peligros relacionados al agua, impartir clases de natación, dejar solos en la bañadera a niños menores de tres años o sobre el uso de casco al andar en bicicleta (C).

Lesiones traumáticas vehiculares. Consejo preventivo a pacientes y padres de niños jóvenes (B)

Los accidentes de tránsito son la primera causa de muerte entre los 18 y 39 años. Se recomienda el uso de cinturones de seguridad ( $y$ asientos de seguridad para niños) para todos los ocupantes de un vehículo; usar casco al andar en moto o bicicleta y evitar conducir bajo los efectos de alcohol o drogas.

\section{Tabaquismo}

Consejo de cese tabáquico en adultos y embarazadas (A) Existe buena evidencia de que el cese tabáquico reduce el riesgo de enfermedad coronaria, accidente cerebrovascular y enfermedad pulmonar. Existe también evidencia adecuada que apoya la efectividad de intervenciones breves de cese tabáquico en adultos en ámbitos de atención primaria. Estas incluyen: rastreo, consejo conductual breve (menor a diez 10 minutos e incluso, aunque menos efectivas, menores a tres minutos) y farmacoterapia; e incrementan el número de personas que abandonan el tabaquismo y se mantienen abstinentes al año. El marco de consejo conductual de las "Cinco A" proporciona una estrategia útil para involucrar a los pacientes en discusiones sobre dejar de fumar:

1) "Ask": preguntar sobre el consumo de tabaco; 2) "Advise": asesorar a dejar a través de claros mensajes personalizados; 3) "Assess": evaluar la voluntad de dejar de fumar, 4) "Assist": ayudar a dejar de fumar y 5) "Arrange": organizar el seguimiento y apoyo.

Reducir el tabaquismo durante el embarazo tiene importantes beneficios en salud tanto para el bebé como para la madre. Si bien existe aun insuficiente evidencia como para evaluar la seguridad o la eficacia del tratamiento farmacológico durante el embarazo, si existe evidencia adecuada que, comparado con intervenciones genéricas breves, la aplicación de intervenciones extendidas de cese tabáquico (cinco a 15 minutos, usando mensajes y materiales de autoayuda específicos) dirigidas a fumadoras embarazadas incrementan substancialmente la tasa de abstinencia durante el embarazo e incrementa el peso de los neonatos. Se concluyó que hay buena evidencia indirecta de que aún pequeños aumentos de las tasas de abandono debidas al consejo producirían importantes beneficios en salud por lo que los beneficios del consejo superan holgadamente los daños potenciales.

\section{Consejo preventivo o de cese tabáquico en niños y adoles-} centes (I)

Se hallo evidencia limitada de que el rastreo y consejo en niños y adolescentes en ámbitos de atención primaria sean efectivos previniendo el inicio o promoviendo el abandono del tabaco. Por lo tanto, no puede determinarse el balance entre beneficios y daños de las intervenciones preventivas o de cese antitabáquico en ámbitos clínicos en niños y adolescentes.

\section{Enfermedades de transmisión sexual}

Consejo en adolescentes sexualmente activos $y$ adultos con riesgo incrementado (B)

En poblaciones con altas tasas de ETS, los pacientes sexualmente activos involucrados en relaciones no monógamas 
pueden considerarse de riesgo. Una ETS en el último año, o múltiples parejas sexuales también implican un mayor riesgo. Existe evidencia aceptable de que el consejo conductual de alta intensidad dirigido a adolescentes sexualmente activos y adultos en mayor riesgo reducen la incidencia de ETS luego de 6 a 12 meses de la consejería. La intervención tiene un beneficio neto moderado en estas poblaciones.

Consejo en adolescentes sexualmente inactivos y adultos sin riesgo incrementado (l)

Faltan estudios sobre el comportamiento de la consejería dirigidas a los adultos que no presentan un riesgo mayor para las ETS y en adolescentes sexualmente inactivos. Existe insuficiente evidencia como para recomendar a favor o en contra del consejo médico en estas poblaciones.

\section{Otras condiciones}

Consejo de actividad física en atención primaria (I)

Existe evidencia suficiente para recomendar la práctica de actividad física de moderada intensidad (caminatas, andar despacio en bicicleta, baile, etc.) en favor de reducir la mortalidad por todas las causas, incidencia de enfermedad coronaria, diabetes no insulinodependiente e hipertensión arterial (B). Sin embargo, se encontró escasa evidencia como para determinar si en ámbitos de atención primaria el consejo que promueva dicha actividad física en pacientes adultos conduce a su cometido.

\section{Embarazo no deseado. Consejo a hombres y mujeres en riesgo de sufrirlo (B)}

El consejo periódico sobre métodos anticonceptivos eficaces se recomienda a mujeres y hombres en riesgo de sufrir un embarazo no deseado. El consejo debe basarse en las preferencias, habilidades y riesgos individuales de cada paciente. Los pacientes sexualmente activos también deben recibir la información sobre medidas para prevenir las enfermedades de transmisión sexual (ETS).

\section{Violencia juvenil. Consejo a adolescentes y adultos asin- tomáticos (I)}

No existe suficiente evidencia como para recomendar a favor o en contra sobre el consejo médico dirigido a adolescentes y adultos asintomáticos para prevenir la morbimortalidad por la violencia juvenil. Sin embargo, los médicos deben estar alerta a los síntomas y señales del uso de drogas, de las varias pre- sentaciones de violencia familiar y de ideación suicida en las personas con factores de riesgo establecidos.

\section{Llanto nocturno. Consejo anticipatorio para padres (A)}

El despertar nocturno con llanto ocurre en al menos el $20 \%$ de los niños luego de la edad en la que estos requieren alimentarse durante la noche. La evidencia demostró que el consejo reduce la prevalencia de este problema. Existe buena evidencia para recomendar a los padres ignorar el llanto nocturno, si este problema es detectado en la visita de los seis meses y produce estrés en los padres.

Dolor de espalda en adultos. Consejo sobre intervenciones preventivas en adultos en atención primaria (I)

No se halló evidencia de buena calidad sobre la efectividad de los ejercicios de fortalecimiento de espalda, o sobre la modificación de sus factores de riesgo asociados (ej. aumento de actividad física, cese tabáquico o reducción del consumo de alcohol) para la prevención de la lumbalgia en adultos. Por otro lado, existe evidencia limitada de la efectividad a corto plazo de sesiones educacionales provistas en ámbitos laborales (ej. escuelas de espalda) dirigidas a pacientes con dolor crónico o recurrente. Existe insuficiente evidencia para recomendar el uso de fajas en el trabajo; incluso algunas intervenciones, como soportes mecánicos, pueden aumentar el riesgo de dolor. No puede determinarse entonces el equilibrio entre beneficios y daños de las distintas intervenciones preventivas relacionadas al dolor de espalda.

Promoción de la lactancia durante y después del embarazo (B) Las intervenciones pueden incluir a la pareja u otros miembros de la familia y amigos. Pueden implicar múltiples estrategias, tales como la educación formal de la lactancia materna, el apoyo directo de las madres durante observaciones de la lactancia, y la formación de personal profesional de la salud sobre la lactancia materna y sobre técnicas de apoyo a la lactancia. La evidencia sugiere que las intervenciones que incluyen tanto atención prenatal como postnatal pueden ser la forma más eficaz para aumentar la duración de la lactancia materna. Se exceptúa de la recomendación a madres con infección por el virus de la inmunodeficiencia humana (HIV) y los niños con galactosemia. Existe evidencia aceptable que apoya las intervenciones de promoción de la lactancia, asociándose a éstas un moderado beneficio neto consistente en una mayor tasa de inicio, mantenimiento y exclusividad de la lactancia.

Ver glosario*

Recibido el 20/05/2009 y aceptado el 20/06/2009

\section{Referencias}

1. Ciapponi A. Guía de Práctica Clínica Actualizaciones de cuidados preventivos de la USPSTF (3 ra edición, 2000-2003) Disponible en: http://www.foroaps.org/hitalba-pagina-articulo.php?cod_producto=956

2. Ciapponi A. Nueva guía de cuidados preventivos de la Fuerza de Tareas de Estados Unidos (primera entrega). Evid actual pract ambul 2005;8:179-182. Disponible en: http://www.foroaps.org/files/nueva\%20fuerza.pdf

3. Adaptado por Ciapponi A de la. Nueva guía de cuidados preventivos de la Fuerza de Tareas de Estados Unidos (segunda entrega). Evid. actual. práct. ambul; 9(1): 26-30, Ene-Feb 2006. Disponible en: http://www.foroaps.org/files/cuida\%20preven\%202\%20parte.pdf

4. U.S. Preventive Services Task Force Grade Definitions. May 2008. Agency for Healthcare Research and Quality, Rockville, MD. Disponible en: http://www.ahrq.gov/clinic/uspstf/grades.htm

5. Atkins D y col. GRADE Working Group. Grading quality of evidence and strength of recommendations. BMJ. 2004;328:1490. Disponible en español en: http://www.gradeworkinggroup.org/_ES/publications/Traduccion_GRADE-BMJ-Atencion_Primaria.pdf

6. Marzo Castillejo M y col. ¿Cómo clasificar la calidad de la evidencia y la fuerza de las recomendaciones?[Editorial] Atención primaria 37:11, 5-8, 2006. Disponible en: http://www.gradeworkinggroup.org/_ES/publications/Editorial_Atencion_Primaria_GRADE.pdf

7. Alonso P y col. El sistema GRADE para la evaluación de la calidad de la evidencia y la graduación de la fuerza de las recomendaciones (en línea). Guías Clínicas $2008 ; 8$ (42). Disponible en: www.msp.gub.uy/andocasociado.aspx?1978,14857 